\title{
Drude weight and optical conductivity of a two-dimensional heavy-hole gas with $k$-cubic spin-orbit interactions
}

\author{
Alestin Mawrie and Tarun Kanti Ghosh \\ Department of Physics, Indian Institute of Technology-Kanpur, Kanpur-208 016, India
}

(Dated: October 8, 2018)

\begin{abstract}
We present detailed theoretical study on zero-frequency Drude weight and optical conductivity of a two-dimensional heavy-hole gas $(2 \mathrm{DHG})$ with $k$-cubic Rashba and Dresselhaus spin-orbit interactions. The presence of $k$-cubic spin-orbit couplings strongly modifies the Drude weight in comparison to the electron gas with $k$-linear spin-orbit couplings. For large hole density and strong $k$-cubic spin-orbit couplings, the density dependence of Drude weight deviates from the linear behavior. We establish a relation between optical conductivity and the Berry connection. Unlike two-dimensional electron gas with $k$-linear spin-orbit couplings, we explicitly show that the optical conductivity does not vanish even for equal strength of the two spin-orbit couplings. We attribute this fact to the non-zero Berry phase for equal strength of $k$-cubic spin-orbit couplings. The least photon energy needed to set in the optical transition in hole gas is one order of magnitude smaller than that of electron gas. Types of two van Hove singularities appear in the optical spectrum are also discussed.
\end{abstract}

PACS numbers: 78.67.-n, 72.20.-i, 71.70.Ej

\section{INTRODUCTION}

Spin-orbit coupling ${ }^{1-4} \underline{\underline{4}}$ plays a vital role in several physical properties of various systems because it breaks the spin degeneracy even at zero magnetic field. There are mainly two kinds of spin-orbit interaction in condensed matter systems, namely Rashba spin-orbit coupling due to inversion asymmetry of the confining potential $1^{5.6}$ and Dresselhaus spin-orbit coupling generated by the asymmetry of the host bulk crystals $-\frac{9}{-1}$. The Rashba spinorbit coupling has been realized in various systems such as zincblende semiconductor quantum wells $\underline{\underline{10}}$, carbon nanotubes $\frac{11}{1}$, two-dimensional materials $\frac{12,13}{415}$, and neutral atomic Bose-Einstein condensates ${ }^{14}, 15$. The spinorbit interaction (SOI) is essential in controlling the spin degree of freedom of the charge carrier in spin-based devices. In most of the studies systems with $k$-linear Rashba and Dresselhaus spin-orbit interactions(RSOI and DSOI) are of much interest. However higher order momentum-dependent spin-orbit interactions have also been seen to dominate in many physical systems. For example, $k$-cubic RSOI dominates in two-dimensional heavy-hole gas formed at the p-type GaAs/AlGaAs heterostructure $\underline{16}-18$, in two-dimensional electron gas formed at the surface of the inversion symmetric oxide $\mathrm{SrTiO}_{3}{ }^{19}$ and in strained $\mathrm{Ge} / \mathrm{SiGe}$ quantum wells $\underline{20}$.

The optical conductivity is due to the transitions from one energy level to another energy level, whereas the zero-frequency Drude weight is associated with the intralevel transitions. The real part of the complex frequencydependent longitudinal optical conductivity provides the absorption as a function of photon energy. Its measurement through optical spectroscopy is an important tool for probing shape of the spin-split energy levels. Typical order of the spin-split energy is the same as that of an electromagnetic radiation with terahertz $(\mathrm{THz})$ frequency. The high-frequency radiation plays an important role to control the spinors of the spin-split energy levels due to the SOIs. Optical response studies will be useful for the high-speed electronic devices since the radiation with $\mathrm{THz}$ frequency flips the spin in a very short time. It opens the possibility of seeing the resonance effects through the optical transition between spin-split levels and leads to unique spectral features.

Several theoretical studies of the optical conductivity have been carried out on spin-orbit coupled electron systems formed at the semiconductor heterojunctions 21,22 as well as in $t_{2 g}$ bands of an oxide with perovskite structure 23 . The optical spectrum of hole gas with $k$ cubic RSOI has been studied 24 partially. The connection between optical longitudinal charge conductivity and optical spin Hall conductivity in electron and hole systems have been established in Refs $\underset{22}{22}$. In Ref $\frac{26}{2}$, it is shown that the optical conductivity disappears when the linear DSOI is same as the RSOI of two-dimensional electron systems. This optical conductivity has also been studied in various single layer two-dimensional materials like graphene ${ }^{27}, \mathrm{MoS}_{2} \frac{28}{}$, silicene $\frac{29}{}$ and surface states of topological insulators 30 .

In this work we present zero-frequency Drude weight and optical conductivity of a two-dimensional heavy-hole gas with $k$-cubic RSOI and DSOI. The Drude weight is strongly modified due to the presence of the $k$-cubic spinorbit couplings. We obtain an analytical expression of the Drude weight when only the $k$-cubic RSOI is present. It deviates from the linear density dependence for large hole density and for strong spin-orbit couplings. It decreases with the increase of the spin-orbit couplings. We show that the optical conductivity is directly related to the Berry connection and does not vanish even for equal strength of the two spin-orbit couplings. The minimum photon energy required for the onset of the optical transition in hole gas is one order of magnitude smaller than that of electron gas. We also identify the nature of the 
two van Hove singularities appear in the optical spectrum.

We organise this paper as follows. In section II, we present basic ground state properties of the heavy-hole system with the $k$-cubic RSOI and DSOI. In section III, we present the effect of spin-orbit interactions on the Drude weight and study various aspects of the optical conductivity for the same system at zero as well as nonzero temperature. Types of the van Hove singularities are tabulated in this section. A summary of our main results are provided in section IV.

\section{PHYSICAL SYSTEM}

The Hamiltonian of a heavy-hole with the both $k$-cubic RSOI and DSOI is given by $31-33$

$$
\begin{aligned}
H & =\frac{\mathbf{p}^{2}}{2 m^{*}}+\frac{i \alpha}{2 \hbar^{3}}\left(p_{-}^{3} \sigma_{+}-p_{+}^{3} \sigma_{-}\right) \\
& -\frac{\beta}{2 \hbar^{3}}\left(p_{-} p_{+} p_{-} \sigma_{+}+p_{+} p_{-} p_{+} \sigma_{-}\right)
\end{aligned}
$$

where $m^{*}$ is the effective mass of the hole, $p_{ \pm}=p_{x} \pm i p_{y}$ and $\sigma_{ \pm}=\sigma_{x} \pm i \sigma_{y}$, with $\sigma_{x}$ and $\sigma_{y}$ are the Pauli's matrices. Also, $\alpha$ is the strength of RSOI which measures the structure inversion asymmetry-induced splitting and $\beta$ is the strength of DSOI which measures the bulk inversion asymmetry-induced spin splitting in the system. Typical value of Rashba strength in narrow gap semiconductor is $\alpha \sim 10^{-22} \mathrm{eV}-\mathrm{cm}^{3}$ and $\beta$ is always less than $\alpha$.

The energy spectrum and the corresponding eigenstates are given by

$$
E_{\lambda}(\mathbf{k})=\frac{\hbar^{2} k^{2}}{2 m^{*}}+\lambda k^{3} \Delta(\theta)
$$

and

$$
\Psi_{\mathbf{k}}^{\lambda}(\mathbf{r})=\frac{\exp (i \mathbf{k} \cdot \mathbf{r})}{\sqrt{2 \Omega}}\left(\begin{array}{c}
1 \\
\lambda e^{i(2 \theta-\phi)}
\end{array}\right)
$$

where $\lambda= \pm$ denotes spin-split energy levels, $\Omega$ is the surface area of the two-dimensional system, and $\Delta(\theta)=\sqrt{\alpha^{2}+\beta^{2}-2 \alpha \beta \sin 2 \theta}$ is the angular anisotropic term with $\theta=\tan ^{-1}\left(k_{y} / k_{x}\right)$ and $\phi=\tan ^{-1}(\alpha \cos \theta-$ $\beta \sin \theta) /(\alpha \sin \theta-\beta \cos \theta)$. The presence of both the SOIs is responsible for the angular anisotropy of the energy spectra. The spin splitting energy $E_{g}(\mathbf{k})=$ $E_{+}(\mathbf{k})-E_{-}(\mathbf{k})=2 k^{3} \Delta(\theta)$ between two branches is also anisotropic. The maximum and minimum spin splitting occurs at $\theta=3 \pi / 4$ or $7 \pi / 4$ and $\theta=\pi / 4$ or $5 \pi / 4$; and the corresponding spin splitting energy values are $E_{g}=2 k^{3}(\alpha \pm \beta)$, respectively.

The Berry connection for this system is given by

$$
\mathbf{A}_{\mathbf{k}}=i\left\langle\psi_{\mathbf{k}}^{\lambda}\left|\nabla_{\mathbf{k}}\right| \psi_{\mathbf{k}}^{\lambda}\right\rangle=\left(\frac{3 \alpha^{2}+\beta^{2}-4 \alpha \beta \sin 2 \theta}{\Delta^{2}(\theta)}\right) \frac{\hat{\theta}}{k}
$$

where $\hat{\theta}=-\sin \theta \hat{x}+\cos \theta \hat{y}$ is the unit polar vector. The corresponding Berry phase is given as

$$
\gamma=\oint \mathbf{A}_{\mathbf{k}} \cdot \mathbf{d k}=\pi+2 \pi \frac{\alpha^{2}-\beta^{2}}{\left|\alpha^{2}-\beta^{2}\right|} .
$$

The Berry connection and Berry phase do not vanish for $\alpha=\beta$ case, in complete contrast to the electron gas with equal strength of the spin-orbit couplings 26.30 .

First we shall calculate density of states (DOS) of the spin-split energy branches, required for the calculation of the Fermi energy $\left(E_{f}\right)$ and the associated anisotropic Fermi wave vectors $k_{f}^{\lambda}(\theta)$. The density of states of the spin-split energy branches are obtained from

$$
\begin{aligned}
D_{\lambda}(E) & =\frac{1}{(2 \pi)^{2}} \int d^{2} k \delta\left(E-E_{\lambda}(\mathbf{k})\right) \\
& =\frac{D_{0}}{2 \pi} \int d^{2} k \frac{\delta\left(k-k_{E}^{\lambda}(\theta)\right)}{\left|k_{E}^{\lambda}(\theta)+\lambda 6 \pi D_{0} \Delta(\theta)\left(k_{E}^{\lambda}(\theta)\right)^{2}\right|}(6)
\end{aligned}
$$

where $D_{0}=m^{*} /\left(2 \pi \hbar^{2}\right)$ is the DOS of spin-polarized hole gas without SOIs and $k_{E}^{\lambda}$ is the real solution of the cubic equation $\left(\hbar k_{E}^{\lambda}\right)^{2} / 2 m^{*}+\lambda \Delta(\theta)\left(k_{E}^{\lambda}\right)^{3}-E=0$. The energy dependence of the density of states is shown in Fig. 1 for two different values of SOIs. The DOS of the heavy-hole spin-split energy branches change asymmetrically with respect to $D_{0}$, whereas it changes symmetrically with respect to $D_{0}$ for $k$-linear spin-orbit coupled electron systems 1 .

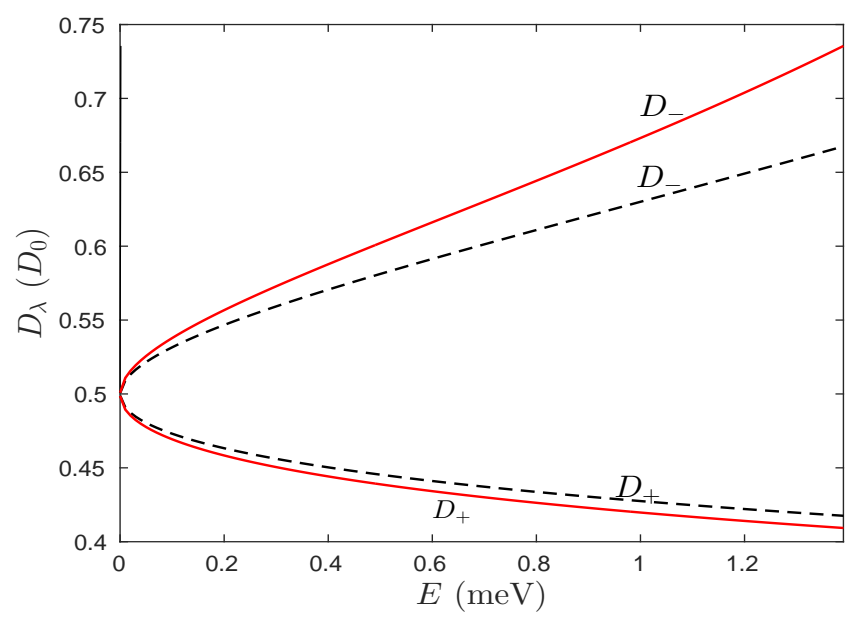

FIG. 1: (color online) Plots of $D_{ \pm}(E)$ (in units of $D_{0}$ ) vs energy $E$ for $\alpha=0.08 \mathrm{eV} \mathrm{nm}^{3}$ (dashed) and $\alpha=0.1 \mathrm{eV} \mathrm{nm}^{3}$ (solid) with $\beta=0.6 \alpha$.

For a given set of system parameters $\left(n_{h}\right.$ being the heavy-hole density, $\alpha$ and $\beta$ ), the Fermi energy $E_{f}$ can be evaluated numerically from the normalization condition: $n_{h}=\sum_{\lambda} \int_{0}^{E_{f}} D_{\lambda}(E) d E$.

For $\beta=0$, the exact analytical expressions of the Fermi wave vectors ${ }^{34}$ are given by $k_{f}^{0, \pm}=\sqrt{3 \pi n_{h}-L /\left(8 l_{\alpha}^{2}\right)} \mp$ 
$L /\left(4 l_{\alpha}\right)$, where $L=\left(1-\sqrt{1-16 \pi n_{h} l_{\alpha}^{2}}\right)$ and $l_{\alpha}=$ $m^{*} \alpha / \hbar^{2}$. When $\beta \neq 0$, it is not possible, due to the anisotropic nature of the spectrum, to derive exact analytical expressions of the spin-split anisotropic Fermi wave vectors $k_{f}^{\lambda}(\theta)$. Therefore, we numerically calculate $k_{f}^{\lambda}(\theta)$ from the following cubic equation: $(\hbar k)^{2} / 2 m^{*}+$ $\lambda k^{3} \Delta(\theta)-E_{f}=0$, for given values of $\alpha, \beta, n_{h}$ and $E_{f}$. The anisotropic Fermi contours $k_{f}^{\lambda}(\theta)$, shown in Fig. 6, are symmetric with respect to the lines $k_{y} \pm k_{x}=0$.

\section{DRUDE WEIGHT AND OPTICAL CONDUCTIVITY}

Consider a two-band system of charge carriers, electron/hole, subjected to an oscillating electric field $(\mathbf{E} \sim$ $\left.\hat{\mathbf{x}} E_{0} e^{i \omega t}\right)$. The complex charge current conductivity is given by

$$
\Sigma_{x x}(\omega)=\sigma_{D}(\omega)+\sigma_{x x}(\omega),
$$

where $\sigma_{D}(\omega)=\sigma_{d} /(1-i \omega \tau)$ is the dynamic Drude conductivity due to the intra-band transitions, with $\sigma_{d}$ being the static Drude conductivity and $\sigma_{x x}(\omega)$ is the complex optical conductivity due to the inter-band optical transitions between two branches. Also, $\tau$ is the momentum relaxation time. It is to be noted here that real part of $\sigma_{D}$ and $\sigma_{x x}$ correspond to the absorptive parts of the optical transition. It implies that absorption peaks in real parts of the conductivities will display dips in the experimentally measured transmission.

The real part of the Drude conductivity is $\operatorname{Re} \sigma_{D}(\omega)=$ $D_{w} \delta(\omega)$, where $D_{w}=\pi \sigma_{d} / \tau$ is known as Drude weight. It shows that the peak appears around $\omega=0$. On the other hand, $\operatorname{Re} \sigma_{x x}(\omega)$ is a function of photon energy with vanishing momentum $(q \rightarrow 0)$. Here the vanishing momentum limit displays the fact that the momentum of the charge carrier is not altered by the electron-photon interaction. An optical absorption occurs through interband transition from $\lambda=-$ branch to $\lambda=+$ branch and helps to make spin-flip transition from one spin branch to another spin branch.

\section{A. Drude weight}

Using the semi-classical Boltzmann transport theory ${ }^{35}$, the Drude weight at very low temperature can be written as

$$
D_{w}=\frac{e^{2}}{4 \pi} \sum_{\lambda} \int d^{2} k\left\langle\hat{v}_{x}\right\rangle_{\lambda}^{2} \delta\left(E_{\lambda}(\mathbf{k})-E_{f}\right)
$$

where $\hat{v}_{x}$ is the $x$-component of the velocity operator and $E_{f}$ is the Fermi energy for a given system. Using Eq. (77), we have calculated (see Appendix for detail calculation) the Drude weight $\left(D_{w}^{e}\right)$ of a two-dimensional electron gas with $k$-linear RSOI, and it is given by

$$
D_{w}^{e}=\frac{\pi e^{2}}{m_{e}}\left(n_{e}-\frac{\alpha_{e}^{2} m_{e}^{2}}{2 \pi \hbar^{4}}\right) .
$$

Here $m_{e}$ is the effective mass of an electron, $\alpha_{e}$ is the spinorbit coupling strength and $n_{e}$ is the density of electrons. This result exactly matches with the result obtained in Ref $\underline{36}$.

For the present problem, the $x$-component of the velocity operator is given by

$$
\hat{v}_{x}=v_{x} I+V_{1} \sigma_{x}-V_{2} \sigma_{y}
$$

where $v_{x}=v \cos \theta, V_{1}=3 v_{\alpha} \sin 2 \theta-v_{\beta}(2+\cos 2 \theta)$, $V_{2}=3 v_{\alpha} \cos 2 \theta+v_{\beta} \sin 2 \theta$, with $v=\hbar k / m, v_{\alpha}=\alpha k^{2} / \hbar$ and $v_{\beta}=\beta k^{2} / \hbar$. Also, $\left\langle\hat{v}_{x}\right\rangle_{\lambda}$ is the average value of the operator $\hat{v}_{x}$ with respect to the state $\psi_{\mathbf{k}}^{\lambda}(\mathbf{r})$. After simplification, it reduces to

$$
D_{w}=\frac{e^{2}}{4 \pi} \sum_{\lambda} \int d \theta \frac{B_{\lambda}(\mathbf{k})}{\left|\hbar^{2} k_{f}^{\lambda} / m^{*}+\lambda 3 \Delta(\theta)\left(k_{f}^{\lambda}\right)^{2}\right|},
$$

where $B_{\lambda}(\mathbf{k})=\left[\left(\hbar k_{f}^{\lambda} / m^{*}\right) \cos \theta+\lambda\left(\left(k_{f}^{\lambda}\right)^{2} / \hbar\right)\{3 \alpha \sin \phi-\right.$ $\beta \cos \phi-2 \beta \cos (2 \theta-\phi)\}]^{2}$. For $\beta=0$, we have the following analytical expression of $D_{w}$ :

$$
D_{w}^{R}=\frac{3 e^{2}}{16 m^{*} l_{\alpha}^{2}}\left[1-\frac{56}{3} \pi n_{h} l_{\alpha}^{2}-\left(1-16 \pi n_{h} l_{\alpha}^{2}\right)^{3 / 2}\right] .
$$

For $\alpha=0$ and $\beta \neq 0$, the Drude weight can be obtained from Eq. (11) by replacing $l_{\alpha}$ by $l_{\beta}=m^{*} \beta / \hbar^{2}$. The variations of $D_{w}$ with $n_{h}$ and $\alpha$ are shown in Fig. 2. It is known that the Drude weight varies linearly with the carrier density for a free fermion as well as for 2DEG with $k$-linear spin-orbit couplings $35-37$. Equation (11) and the left panel of Fig. 2 clearly show deviation from the linear density dependence of $D_{w}$ for large density and strong RSOI. On the other hand, the Drude weight decreases with the increase of the spin-orbit couplings as shown in the right panel of Fig. 2.

It should be mentioned here that the effect of the Coulomb interaction is not taken into account in the above discussion. There may or may not be a significant effect of the Coulomb interaction on $D_{w}$ when the interaction parameter $r_{s}$ is very large (i.e. at the very low densities $n_{h}<0.5 \times 10^{15} \mathrm{~m}^{-2}$ ). For example, the strong effect of the Coulomb interaction in lowdensity 2DHG leads to the negative compressibility observed in Ref $\frac{38}{3}$. On the other hand, based on electron measurements ${ }^{39}$, one would expect an enhanced spin susceptibility at very low densities for 2DHG but it is not observed experimentally $\underline{40}$. The behavior of electrons and holes can be quite different, due to different effective mass and different form of spin-orbit coupling, as revealed in several theoretical studies $41-43$.

\section{B. Optical conductivity}

The Kubo formula for the $x x$ component of the optical conductivity in terms of the Matsubara Green's function 

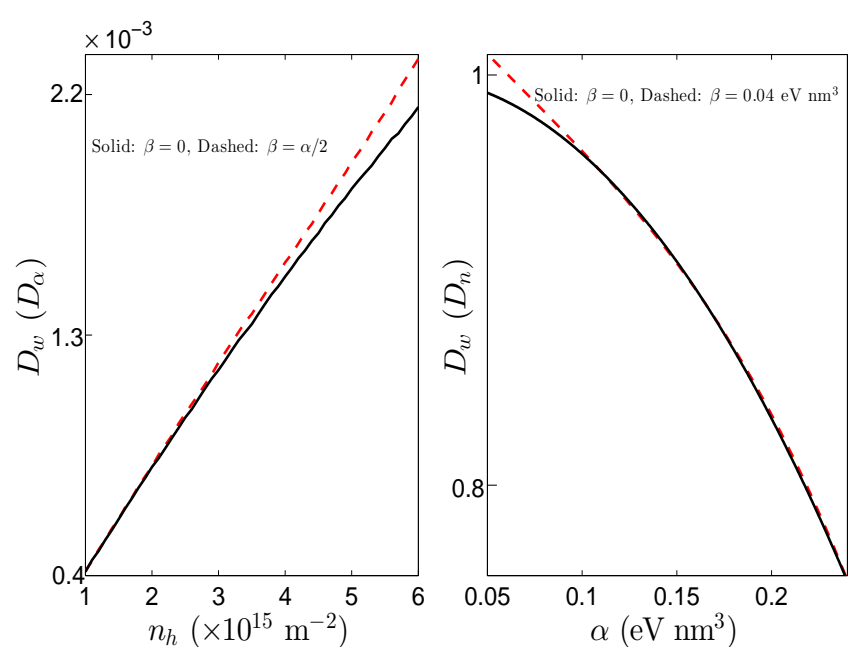

FIG. 2: (color online) Left panel: Plots of $D_{w}$ (in units of $\left.D_{\alpha}=e^{2} \pi / m^{*} l_{\alpha}^{2}\right)$ vs $n_{h}$ for $\alpha=0.12 \mathrm{eV} \mathrm{nm}^{3}$. Right panel: Plots of $D_{w}$ (in units of $D_{n}=e^{2} n_{h} \pi / m^{*}$ ) vs $\alpha$ for $n_{h}=$ $2.0 \times 10^{15} \mathrm{~m}^{-2}$.

is given by

$$
\begin{gathered}
\sigma_{x x}(\omega)=-\frac{e^{2}}{i \omega} \frac{1}{(2 \pi)^{2}} \int_{0}^{\infty} \int_{0}^{2 \pi} k d k d \theta \\
\times T \sum_{l} \operatorname{Tr}\left\langle\hat{v}_{x} \hat{G}\left(\mathbf{k}, \omega_{l}\right) \hat{v}_{x} \hat{G}\left(\mathbf{k}, \omega_{s}+\omega_{l}\right)\right\rangle_{i \omega_{s} \rightarrow \omega+i \delta}
\end{gathered}
$$

Here $T$ is the temperature, $\omega_{s}=(2 s+1) \pi T$ and $\omega_{l}=$ $2 l \pi T$ are the fermion and boson Matsubara frequencies, respectively, with $s$ and $l$ are integers.

The matrix Green's function for the two-level system associated with the Hamiltonian (1) is given by

$$
\begin{aligned}
\hat{G}\left(\mathbf{k}, i \omega_{s}\right) & =\left[i \hbar \omega_{s}+\mu-(\hbar k)^{2} / 2 m^{*}-S_{1} \sigma_{x}-S_{2} \sigma_{y}\right]^{-1} \\
& =\frac{i \hbar \omega_{s}+\mu-\frac{\hbar^{2} k^{2}}{2 m^{*}}+S_{1} \sigma_{x}+S_{2} \sigma_{y}}{\left(i \hbar \omega_{s}+\mu-\frac{\hbar^{2} k^{2}}{2 m^{*}}\right)^{2}-S_{1}^{2}-S_{2}^{2}}
\end{aligned}
$$

with $S_{1}=k^{3}(-\alpha \sin 3 \theta+\beta \cos \theta)$ and $S_{2}=k^{3}(\alpha \cos 3 \theta+$ $\beta \sin \theta$ ). It is convenient to write the Green's function as follows

$$
\hat{G}\left(\mathbf{k}, i \omega_{s}\right)=\frac{1}{2} \sum_{\lambda}[I+\lambda \mathbf{F} \cdot \boldsymbol{\sigma}] G_{0}\left(\mathbf{k}, \lambda, \omega_{s}\right),
$$

where $\mathbf{F}=\left(S_{1}, S_{2}\right) / \sqrt{S_{1}^{2}+S_{2}^{2}}$, and $G_{0}\left(\mathbf{k}, \lambda, \omega_{s}\right)=$ $1 /\left(i \hbar \omega_{s}+\mu-\hbar^{2} k^{2} / 2 m^{*}-\lambda \sqrt{S_{1}^{2}+S_{2}^{2}}\right)$.

Now we can write down $\hat{v}_{x} \hat{G}\left(\mathbf{k}, \omega_{l}\right)$ as below

$$
\hat{v}_{x} \hat{G}\left(\mathbf{k}, \omega_{l}\right)=\frac{1}{2} \sum_{\lambda} M_{\lambda} G_{0}\left(\mathbf{k}, \lambda, \omega_{l}\right)
$$

where $M_{\lambda}=\left(v+V_{1} \sigma_{x}-V_{2} \sigma_{y}\right)(I+\lambda \mathbf{F} \cdot \boldsymbol{\sigma})$, which will give us

$$
\begin{aligned}
& \operatorname{Tr}\left\langle\hat{v}_{x} \hat{G}\left(\mathbf{k}, \omega_{l}\right) \hat{v}_{x} \hat{G}\left(\mathbf{k}, \omega_{s}+\omega_{l}\right)\right\rangle= \\
& \frac{1}{2} \sum_{\lambda \lambda^{\prime}}\left[\left(1+\lambda \lambda^{\prime}\right) v^{2}+V_{1}^{2}+V_{2}^{2}+\lambda \lambda^{\prime}\left\{\left(V_{1} F_{x}-V_{2} F_{y}\right)^{2}\right.\right. \\
& \left.\left.-\left(V_{1} F_{y}+V_{2} F_{x}\right)^{2}\right\}+2\left(\lambda+\lambda^{\prime}\right) v\left(V_{1} F_{x}-V_{2} F_{y}\right)\right] \\
& \times G_{0}\left(\mathbf{k}, \lambda, \omega_{l}\right) G_{0}\left(\mathbf{k}, \lambda^{\prime}, \omega_{s+l}\right) .
\end{aligned}
$$

Using the identity

$$
\begin{aligned}
& T \sum_{l}\left[\frac{1}{\left(i \hbar \omega_{l}+\mu-E_{\lambda}\right)} \cdot \frac{1}{i \hbar\left(\omega_{s}+\omega_{l}\right)+\mu-E_{\lambda^{\prime}}}\right] \\
= & \begin{cases}\frac{f\left(E_{\lambda}\right)-f\left(E_{\lambda^{\prime}}\right)}{i \hbar \omega_{s}-E_{\lambda^{\prime}}+E_{\lambda}} & \lambda \neq \lambda^{\prime} \\
0 & \lambda=\lambda^{\prime}\end{cases}
\end{aligned}
$$

with the Fermi-Dirac distribution function $f(E)=$ $\left[e^{(E-\mu) /\left(k_{B} T\right)}+1\right]^{-1}$, one can see that the contribution of the intraband transition $\left(\lambda=\lambda^{\prime}\right)$ to the optical conductivity is simply zero. This happens as a result of the momentum conservation. The non-zero contribution is coming only from the interband transitions $\left(\lambda \neq \lambda^{\prime}\right)$. With this we can simplify further as

$$
\begin{aligned}
& T \sum_{l} \operatorname{Tr}\left\langle\hat{v}_{x} \hat{G}\left(\mathbf{k}, \omega_{l}\right) \hat{v}_{x} \hat{G}\left(\mathbf{k}, \omega_{s}+\omega_{l}\right)\right\rangle \\
= & \frac{\left(S_{2} V_{1}+S_{1} V_{2}\right)^{2}}{S_{1}^{2}+S_{2}^{2}}\left[\frac{f\left(E_{-}\right)-f\left(E_{+}\right)}{i \hbar \omega_{s}-E_{+}+E_{-}}+\left(E_{-} \leftrightarrow E_{+}\right)\right],
\end{aligned}
$$

thereby we obtain the expression for the optical conductivity as follows

$$
\begin{aligned}
\sigma_{x x}(\omega)= & -\frac{e^{2}}{i(2 \pi \hbar)^{2} \omega} \int_{0}^{\infty} \int_{0}^{2 \pi} k^{5} H(\theta) d k d \theta \\
& \times\left[\frac{f\left(E_{-}\right)-f\left(E_{+}\right)}{\hbar \omega+i \delta-E_{+}+E_{-}}+\left(E_{-} \leftrightarrow E_{+}\right)\right](17)
\end{aligned}
$$

where the explicit expression of the optical matrix element $H(\theta)$ is given by $H(\theta)=\sin ^{2} \theta\left(3 \alpha^{2}+\beta^{2}-\right.$ $4 \alpha \beta \sin 2 \theta)^{2} / \Delta^{2}(\theta)$. It is interesting to note that the above equation can be re-written in terms of the $x$ component of the Berry connection $\left(A_{k_{x}}\right)$, which is given by

$$
\begin{aligned}
\sigma_{x x}(\omega) & =-\frac{e^{2}}{i(2 \pi \hbar)^{2} \omega} \int_{0}^{\infty} \int_{0}^{2 \pi} k^{7} \Delta^{2}(\theta) A_{k_{x}}^{2} d k d \theta \\
& \times\left[\frac{f\left(E_{-}\right)-f\left(E_{+}\right)}{\hbar \omega+i \delta-E_{+}+E_{-}}+\left(E_{-} \leftrightarrow E_{+}\right)\right] .
\end{aligned}
$$

Similar connection has been established for $\mathrm{MoS}_{2}$ system 28 .

We have also calculated other components of the optical conductivity i.e. $\sigma_{y y}(\omega)$ and $\sigma_{x y}(\omega)$. We find that $\sigma_{y y}(\omega)=\sigma_{x x}(\omega)$ and $\sigma_{x y}(\omega)=0=\sigma_{y x}(\omega)$. It implies that the anisotropic Fermi contours do not lead to 
anisotropic optical conductivity. This is similar to the isotropic charge conductivity of a two-dimensional electron gas with combined RSOI and DSOI ${ }^{37}$. Moreover, $\sigma_{y y}(\omega)$ can also be expressed in terms of the $y$-component of the Berry connection, similar to the $\sigma_{x x}(\omega)$ case.

Keeping in mind that $\omega>0$ the absorptive part of the optical conductivity $\sigma_{x x}(\omega)$ simplifies to

$$
\begin{aligned}
\operatorname{Re}\left[\sigma_{x x}(\omega)\right] & =\frac{e^{2}}{4 \pi \hbar} \frac{1}{\hbar \omega} \int_{0}^{\infty} \int_{0}^{2 \pi} k^{5} H(\theta) d k d \theta \\
& \times\left[f\left(E_{-}\right)-f\left(E_{+}\right)\right] \delta\left(\hbar \omega-2 k^{3} \Delta(\theta)\right) \\
& =\frac{e^{2}}{16 \pi \hbar} \int_{0}^{2 \pi} d \theta \frac{\sin ^{2} \theta\left[3+\eta^{2}-4 \eta \sin 2 \theta\right]^{2}}{3\left[1+\eta^{2}-2 \eta \sin (2 \theta)\right]^{2}} \\
& \times\left[f\left(E_{-}\left(k_{\omega}\right)\right)-f\left(E_{+}\left(k_{\omega}\right)\right)\right],
\end{aligned}
$$

with $k_{\omega}^{3}=\hbar \omega / 2 \Delta(\theta)$ and $\eta=\beta / \alpha$.

Pure Rashba $(\beta=0)$ : In the absence of DSOI $(\beta=$ 0 ), the absorptive part of the optical conductivity at finite temperature is given by

$$
\operatorname{Re}\left[\sigma_{x x}(\omega)\right]=\frac{3 e^{2}}{16 \hbar}\left[f\left(E_{-}\left(k_{\omega}\right)\right)-f\left(E_{+}\left(k_{\omega}\right)\right)\right],
$$

with $k_{\omega}^{3}=\hbar \omega / 2 \alpha$. At zero temperature we have

$$
\operatorname{Re}\left[\sigma_{x x}(\omega)\right]=\frac{3 e^{2}}{16 \hbar}\left[\Theta\left(E_{-}\left(k_{\omega}\right)-\mu\right)-\Theta\left(E_{+}\left(k_{\omega}\right)-\mu\right)\right],
$$

where $\Theta(x)$ is the usual unit step function.

Depending on the carrier density $\left(n_{h}\right)$ and spin-orbit coupling strength $(\alpha)$, there must be an upper and lower limits of the photon energy $\left(E_{p}=\hbar \omega\right)$ in order to have transitions from the initial state $\lambda=-1$ to the final state $\lambda=+1$. We use the following parameters for various plots: charge carrier density $n_{h}=2.4 \times 10^{15} \mathrm{~m}^{-2}$ and heavy hole mass $m=0.41 m_{0}$ with $m_{0}$ is the bare electron mass. In Fig. 3, we plot the optical conductivity $\sigma_{x x}(\omega)$ vs photon energy $E_{p}$ for fixed $\alpha=0.1 \mathrm{eV} \mathrm{nm}^{3}$ at four different temperatures. At $T=0$, the interband transitions take place only when photon energy satisfies the following inequality: $2 \alpha\left(k_{f}^{0,-}\right)^{3} \leq \hbar \omega \leq 2 \alpha\left(k_{f}^{0,+}\right)^{3}$ and the optical conductivity becomes box function with the edges at $E_{\text {edge }}^{ \pm}=2 \alpha\left(k_{f}^{0, \pm}\right)^{3}$. The width of the optical absorption is then $\Delta_{b}=2 \alpha\left[\left(k_{f}^{0,+}\right)^{3}-\left(k_{f}^{0,-}\right)^{3}\right]$, whose variation with $n_{h}$ and $\alpha$ are shown in Fig. 4. At finite temperature, the optical conductivity deviates from the box function and smears beyond the box edges. Moreover, the conductivity at the box edges $\left(E_{\text {edge }}^{ \pm}\right)$is always $\sigma_{0} / 2$ because of the nature of the Fermi distribution function. The peaks in the optical conductivity at finite $T$ is located near the center of the box and it is given by $E_{\text {peak }} \simeq\left(E_{\text {edge }}^{+}+E_{\text {edge }}^{-}\right) / 2=\alpha\left[\left(k_{f}^{0,+}\right)^{3}+\left(k_{f}^{0,-}\right)^{3}\right]$. Figure 4 shows that $\Delta_{b}$ increases with $n_{h}$ and $\alpha$. We mention here that similar analysis can be done for the opposite case i.e. $\alpha=0$ but $\beta \neq 0$. The optical conductivity at zero temperature will be $\sigma(\omega)=e^{2} /(48 \hbar)$ which is 9 times less than $\beta=0$ case. Other results will be the same as for $\alpha \neq 0$ but $\beta=0$ case.

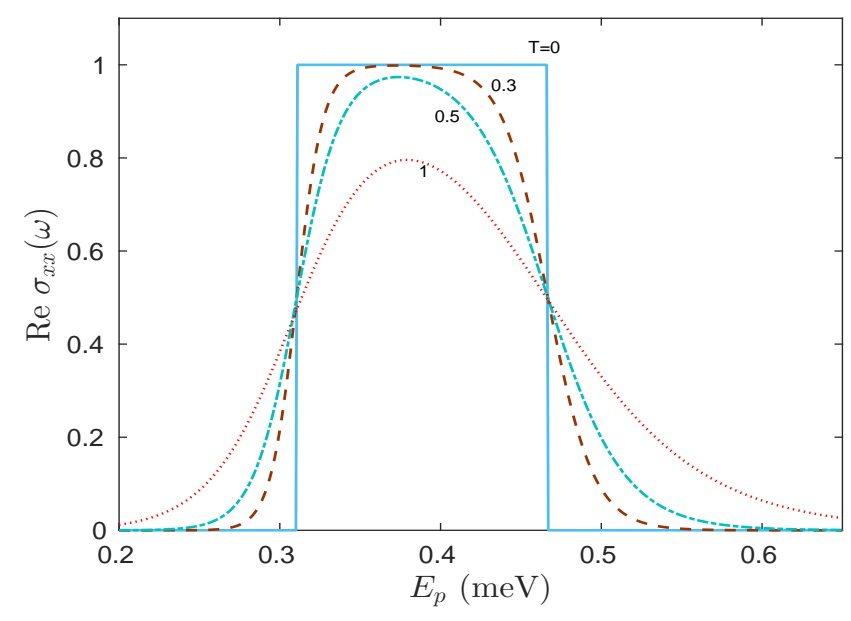

FIG. 3: (color online) Plots of $\sigma(\omega)$ (in units of $\sigma_{0}=3 e^{2} / 16 \hbar$ ) vs $E_{p}$ at four different temperatures.

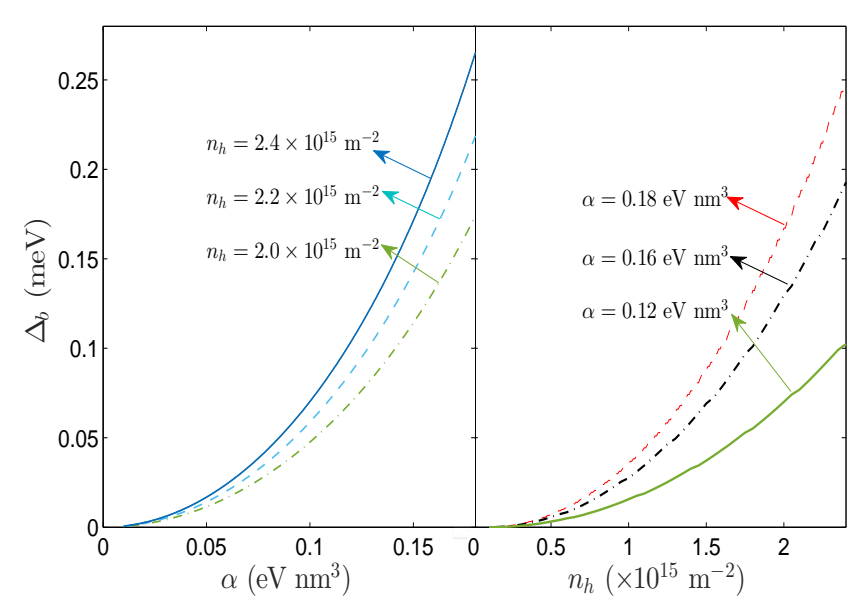

FIG. 4: (color online) (a) Bandwidth $\Delta_{b}$ vs $\alpha$ at various hole density and (b) $\Delta_{b}$ vs $n_{h}$ for various $\alpha$.

Non-zero Rashba and Dresselhaus SOIs: Now we discuss how the simultaneous presence of both the spin-orbit interactions modifies the behavior of the optical conductivity. Similar to the previous case, the optical transitions between the initial state $\lambda=-1$ and the final state $\lambda=+1$ can take place only when photon energy satisfies the following inequality: $\epsilon_{-}(\theta) \leq \hbar \omega \leq$ $\epsilon_{+}(\theta)$ with $\epsilon_{ \pm}=2\left(k_{f}^{ \pm}(\theta)\right)^{3} \Delta(\theta)$. The values of $k_{f}^{ \pm}(\theta)$ are the numerical solutions of the two cubic equations $\left(\hbar k_{f}^{ \pm}\right)^{2} / 2 m^{*} \pm\left(k_{f}^{ \pm}\right)^{3} \Delta(\theta)-E_{f}=0$, where $E_{f}$ is the Fermi energy for given values of $\alpha, \beta$ and $n_{h}$.

In the top panel of Fig. $5, \epsilon_{ \pm}(\theta)$ vs photon energy are plotted. The shaded angular region contribute to the optical transitions. The interband optical conductivity $\sigma_{x x}(\omega)$ vs $E_{p}$ is displayed in the middle panel of Fig. 


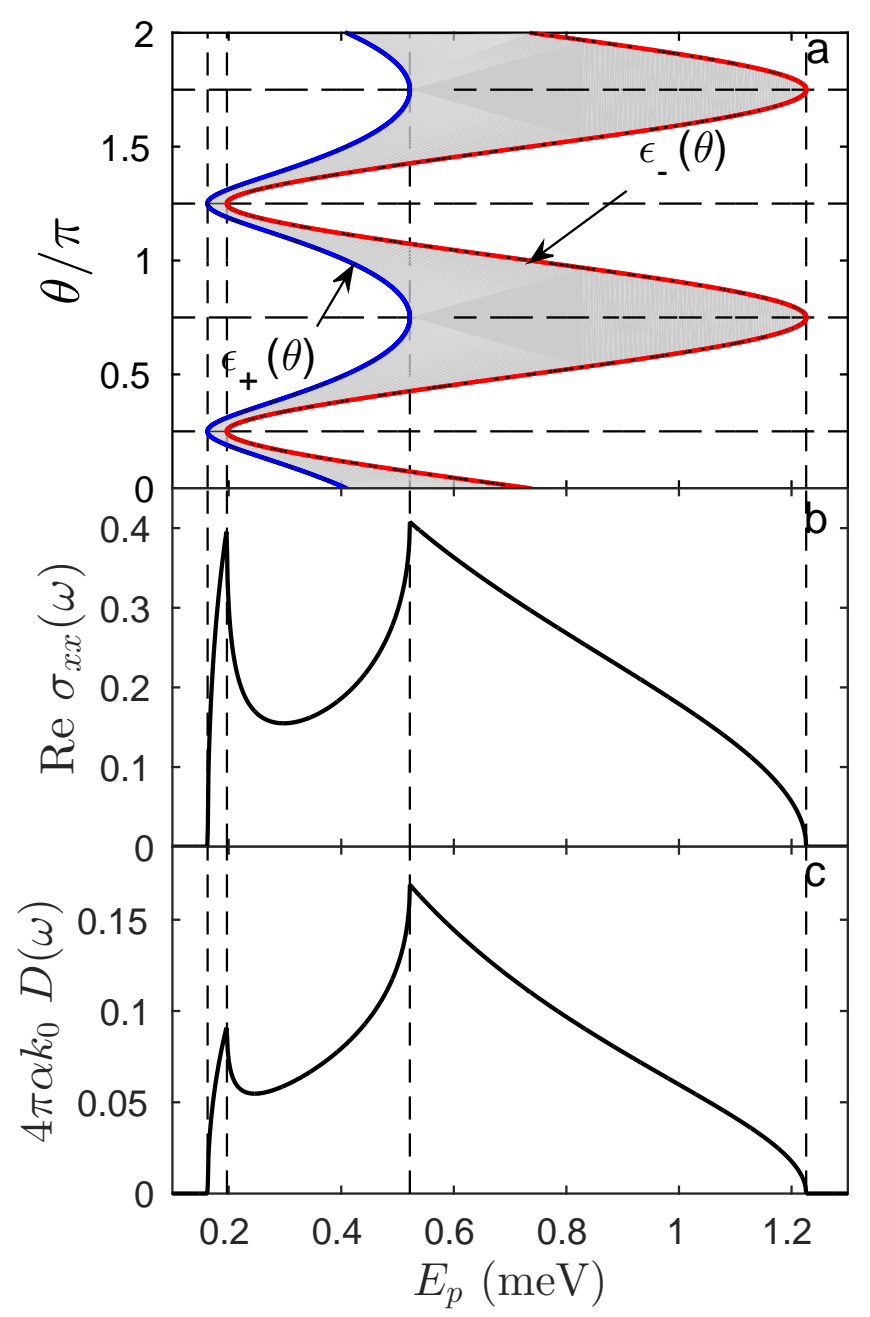

FIG. 5: (color online) Top panel: $\epsilon_{ \pm}=2\left(k_{f}^{ \pm}(\theta)\right)^{3} \Delta(\theta)$, Middle panel: $\sigma_{x x}(\omega)$ (in units of $\sigma_{0}$ ) vs $E_{p}$ and bottom panel: joint density of states $D(\omega)$ with $k_{0}=\sqrt{2 \pi n_{h}}$. Here $\alpha=0.12$ $\mathrm{eV} \mathrm{nm^{3 }}, \eta=0.6$.

5. We see that the optical transition begins and ends at $E_{p}=0.162 \mathrm{meV}$ and $E_{p}=1.226 \mathrm{meV}$, respectively. Looking at the top panel, one can see that these values correspond to $\epsilon_{+}(\pi / 4)=\epsilon_{+}(5 \pi / 4)=\epsilon_{1}$ and $\epsilon_{-}(3 \pi / 4)=$ $\epsilon_{-}(7 \pi / 4)=\epsilon_{4}$, respectively. The minimum (maximum) photon energy $\epsilon_{1}\left(\epsilon_{4}\right)$ needed for interband optical transitions correspond to the excitation of a heavy hole with the Fermi wave vector $k_{f}^{+}(\theta)\left(k_{f}^{-}(\theta)\right)$ at $\theta=\pi / 4$ or $5 \pi / 4$ $(\theta=3 \pi / 4$ or $7 \pi / 4)$. The optical absorption edges of Fig. 5 are exactly $\epsilon_{1}$ and $\epsilon_{4}$. Moreover, two peaks of the optical conductivity occur at $E_{p}=0.196 \mathrm{meV}$ and $E_{p}=0.522$ $\mathrm{meV}$. It is easy to see from the top panel of Fig. 5 that these values correspond to $\epsilon_{-}(\pi / 4)=\epsilon_{-}(5 \pi / 4)=\epsilon_{2}$ and $\epsilon_{+}(3 \pi / 4)=\epsilon_{+}(7 \pi / 4)=\epsilon_{3}$, respectively.

In order to understand these behaviors we plot a constant energy-difference curve $E_{g}(\mathbf{k})=2 \Delta(\theta) k^{3}=\hbar \omega$ for $\hbar \omega=\epsilon_{1}\left(C_{1}\right.$ : dotted-blue $), \hbar \omega=\epsilon_{2}\left(C_{2}\right.$ : solid-blue $)$,

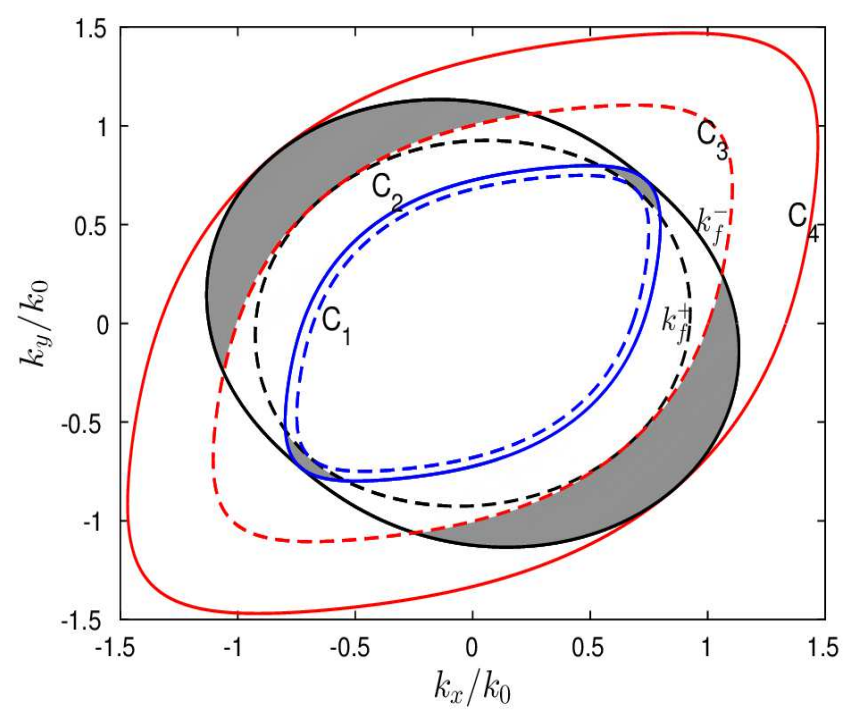

FIG. 6: (color online) This figure shows the Fermi contour $k_{f}^{+}(\theta)$ (dotted-black), $\left.k_{f}^{-}(\theta)\right)$ (solid-black), the constant energy-difference $C(\hbar \omega)=\hbar \omega=2 \Delta(\theta) k^{3}$ for $\hbar \omega=\epsilon_{1}$ (dashed-blue), $\hbar \omega=\epsilon_{4}$ (solid-blue), $\hbar \omega_{2}=\epsilon_{2}$ (dashed-red) and $\hbar \omega_{4}=\epsilon_{3}$ (solid-red).

$\hbar \omega=\epsilon_{3}\left(C_{3}\right.$ : dashed-red $)$ and $\hbar \omega=\epsilon_{4}\left(C_{4}\right.$ : solid-red $)$ in Fig. 6. Because of the angular anisotropy in the dispersion relation, the optical conduction becomes $\mathbf{k}$-selective as shown in the shaded portions of Fig. 6 where the $C_{i}$ 's $(i=1,2,3,4)$ intersect with the two Fermi lines $k_{f}^{+}(\theta)$ (dotted) and $k_{f}^{-}(\theta)$ (solid), respectively.

Two peaks in the optical conductivity can be explained by analyzing the joint density of states. Usually, the absorptive part of the optical conductivity is characterized by the joint density of states between the spin-split branches, which is given by

$$
D(\omega)=\frac{1}{(2 \pi)^{2}} \int d^{2} k\left[f\left(E_{+}\right)-f\left(E_{-}\right)\right] \delta\left(E_{g}(\mathbf{k})-\hbar \omega\right) .
$$

Using the standard approach, we can write the joint DOS as

$$
D(\omega)=\frac{1}{(2 \pi)^{2}} \int \frac{d C\left[f\left(E_{+}\left(k_{\omega}\right)\right)-f\left(E_{-}\left(k_{\omega}\right)\right)\right]}{\left|\partial_{k} E_{g}(\mathbf{k})\right|_{E_{g}=\hbar \omega}},
$$

where $d C$ is the line element along the contour and $k_{\omega}=(\hbar \omega / 2 \Delta(\theta))^{1 / 3}$. The peaks appear in Fig. 5 whenever $\left|\partial_{k} E_{g}(\mathbf{k})\right|$ in the joint DOS attains a minimum value. Therefore, the two peaks correspond to the van Hove singularities in the joint density of states. The first (second) peak is at a photon energy $\hbar \omega_{2}\left(\hbar \omega_{3}\right)$ for which the longer(shorter) axis of the curve $C_{i}$ coincides with the Fermi line $k_{f}^{-}(\theta)\left(k_{f}^{+}(\theta)\right)$. The joint density of states for finite $\eta$ is shown in the bottom panel of Fig. 5. Locations of the two peaks as well as the optical absorption edges 
in the optical conductivity are exactly described by the joint density of states. The asymmetric splitting at the Fermi level along the symmetry axis $k_{y}= \pm k_{x}$ is thus responsible for the peaks at $\epsilon_{2}$ and $\epsilon_{3}$, respectively. The magnitude and the non-symmetric shape of the optical conductivity is controlled by the factor $H(\theta)$.

For two-dimensional systems, van Hove singularities are classified into three types based on the nature of change of the energy gap $E_{g}(\mathbf{k})$ as we go away from the singular points $\underline{44}$. This can be obtained by using the Taylor series expansion of $E_{g}(\mathbf{k})$ around the singular points $\mathbf{k}_{s}$ at which the energy difference attains minimum value. Here the singular points are at $\mathbf{k}_{s}=(k, \pi / 4$ or $5 \pi / 4)$ and $\mathbf{k}_{s}=(k, 3 \pi / 4$ or $7 \pi / 4)$. Expanding $E_{g}(\mathbf{k})$ around $\mathbf{k}_{s}$ as $E_{g}(\mathbf{k})=E_{g}\left(\mathbf{k}_{s}\right)+\sum_{i} b_{i}\left(k_{i}-k_{s i}\right)^{2}$ with $i=x, y$ and the expansion coefficients are $2 b_{i}=\left.\frac{\partial^{2} E_{g}(\mathbf{k})}{\partial k_{i}^{2}}\right|_{\mathbf{k}_{s}}$. The classification of the van Hove singularities are based on how many coefficients $\left(b_{i}\right)$ are negative. For the present system, the coefficients correspond to the expansion about the singular point $\mathbf{k}_{s}=(k, \pi / 4)$ are given by

$$
b_{x}=\alpha k \frac{9(1-\eta)^{2}+12 \eta}{2(1-\eta)}, \quad b_{y}=\alpha k \frac{9(1-\eta)^{2}-4 \eta}{2(1-\eta)}
$$

and the coefficients correspond to the expansion about the singular point $\mathbf{k}_{s}=(k, 3 \pi / 4)$ are

$$
b_{x}=\alpha k \frac{9(1+\eta)^{2}+4 \eta}{2(1+\eta)}, \quad b_{y}=\alpha k \frac{9(1+\eta)^{2}-12 \eta}{2(1+\eta)} .
$$

The type of singularities that arise are summarized in

\begin{tabular}{|c|c|c|c|c|}
\hline $\begin{array}{c}\text { Singular } \\
\text { point }\end{array}$ & $\eta=\beta / \alpha$ & $b_{x}$ & $b_{y}$ & $\begin{array}{c}\text { Type of } \\
\text { singularity }\end{array}$ \\
\hline \multirow{4}{*}{$(k, \pi / 4)$} & \multirow{2}{*}{$\eta<1$} & \multirow{2}{*}{$>0$} & $>0$ for $\eta<\eta_{l}$ & $M_{0}$ \\
\hline & & & $<0$ for $\eta>\eta_{l}$ & $M_{1}$ \\
\hline & \multirow{2}{*}{$\eta>1$} & \multirow{2}{*}{$<0$} & $>0$ for $\eta<\eta_{h}$ & $M_{1}$ \\
\hline & & & $<0$ for $\eta>\eta_{h}$ & $M_{2}$ \\
\hline$(k, 3 \pi / 4)$ & - & $>0$ & $>0$ & $M_{0}$ \\
\hline
\end{tabular}
the table below:

TABLE I: Table showing the type of singularity. Here, $\eta_{l}=$ $(11-2 \sqrt{10}) / 9$ and $\left.\eta_{h}=(11+2 \sqrt{10}) / 9\right)$ are the solutions of the quadratic equation $9 \eta^{2}-20 \eta+9=0$.

The optical conductivity versus $E_{p}$ at different values of $\eta$ at zero temperature is shown in Fig. 7. Similarly, $\sigma(\omega)$ versus $E_{p}$ at different temperatures for a given value of $\eta$ is shown in Fig. 8.

Now we shall point out here the main differences between the electron and heavy hole systems. Unlike twodimensional electron gas with $k$-linear RSOI and DSOI, the optical conductivity of two-dimensional hole gas with $k$-cubic RSOI and DSOI does not vanish for $\alpha=\beta$ case. This is related to non-zero Berry phase of twodimensional hole gas with equal strength of $k$-cubic RSOI

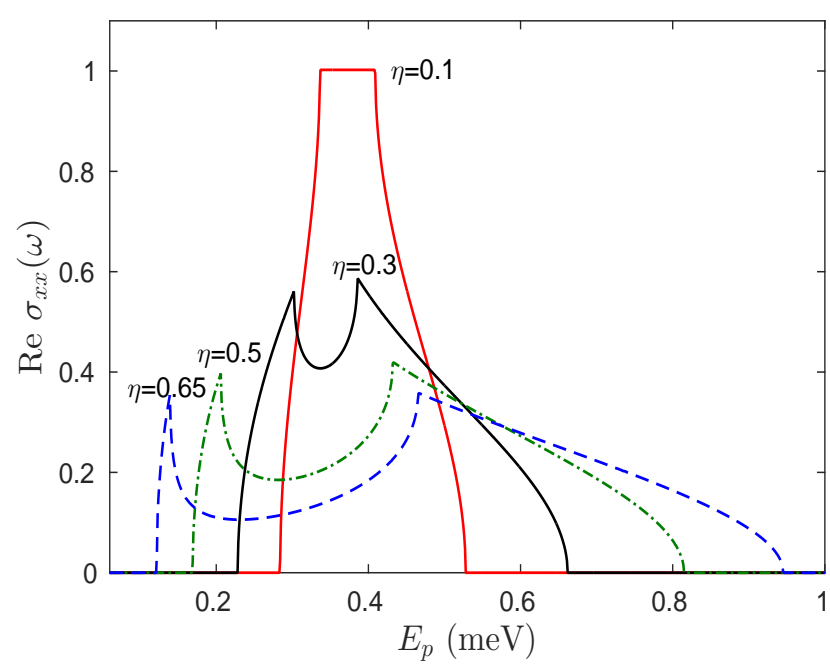

FIG. 7: (color online) Optical conductivity Re $\sigma_{x x}(\omega)$ in units of $\sigma_{0}$ for several values of $\eta$ with $\alpha=0.1 \mathrm{eV} \mathrm{nm}^{3}$.

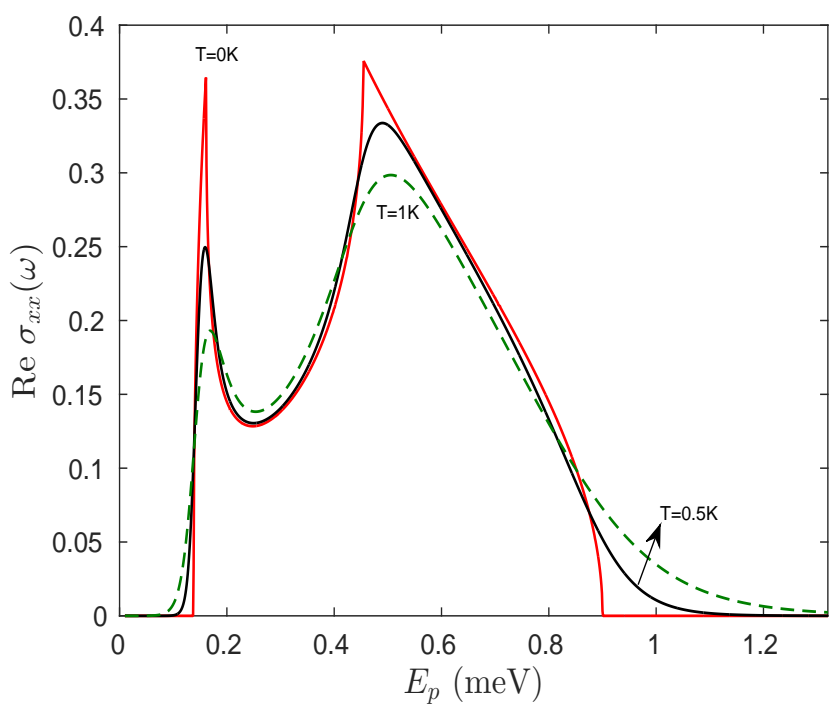

FIG. 8: (color online) Optical conductivity Re $\sigma_{x x}(\omega)$ in units of $\sigma_{0}$ for several values of temperature $T$ with $\alpha=0.1 \mathrm{eV} \mathrm{nm}^{3}$ and $\eta=0.6$.

and DSOI. For realistic systems, the minimum photon energy needed to trigger the optical transition in hole gas is one order of magnitude smaller than that of electron gas. In electron systems, heights of the two peaks are unequal, whereas they are more or less same for hole gas at very low temperature. 


\section{SUMMARY AND CONCLUSION}

We have presented detailed analysis of zero-frequency Drude weight and optical conductivity of a twodimensional heavy-hole gas with $k$-cubic RSOI and DSOI, at both zero and non-zero temperature. We obtained an analytical expression of the Drude weight for Rashba interaction only. It is shown that the Drude weight deviates from the linear density dependence, in contrast to the case of electron gas with and without $k$-linear spin-orbit interactions. It decreases with the increase of the spin-orbit couplings. We have identified a connection between the optical conductivity and the Berry connection. On contray to the electron case, for equal strength of the Rashba and Dresselhaus couplings, the optical conductivity remains finite. This is due to fact that Berry phase is not zero for equal strength of $k$-cubic spin-orbit couplings. The bandwidth increases with increase of the hole density as well as spin-orbit couplings. It is seen that the minimum photon energy required to set in the optical transition in hole gas is one order of magnitude smaller than that of electron gas. We have classified the type of the two van Hove singular points.

\section{Appendix: Calculation of Drude weight for system with linear RSOI}

Here we consider two-dimensional electron gas with $k$-linear spin-orbit interaction and calculate the Drude weight using Eq. (7). The Hamiltonian for this system is given by

$$
H_{e}=\frac{p^{2}}{2 m_{e}}+\frac{\alpha_{e}}{\hbar}\left(\sigma_{x} p_{y}-\sigma_{y} p_{x}\right)
$$

where $m_{e}$ is the electron's effective mass and $\alpha_{e}$ is the strength of the Rashba SOI. The corresponding energy eigenvalues and eigenstates are $E_{\lambda}(\mathbf{k})=\hbar^{2} k^{2} / 2 m_{e}+$ $\lambda \alpha_{e} k$ and $\psi_{\mathbf{k}}^{\lambda}(\mathbf{r})=e^{i \mathbf{k} \cdot \mathbf{r}}\left(1,-i \lambda e^{i \theta}\right)^{T} / \sqrt{2 \Omega}$, respectively. Here $\theta=\tan ^{-1}\left(k_{y} / k_{x}\right)$ and $T$ denotes the transpose operation. The Fermi energy $\left(E_{f}\right)$ can be obtained as $E_{f}=\pi \hbar^{2} n_{e} / m_{e}-m_{e} \alpha_{e}^{2} / \hbar^{2}$ with $n_{e}$ being the electron density.

The $x$-component of the velocity operator is $\hat{v}_{x}=$ $p_{x} / m_{e}-\left(\alpha_{e} / \hbar\right) \sigma_{y}$. Its expectation value is $\left\langle\hat{v}_{x}\right\rangle=$ $\left(\hbar / m_{e}\right)\left(k+\lambda k_{\alpha}\right) \cos \theta$ with $k_{\alpha}=m_{e} \alpha_{e} / \hbar^{2}$.

Following Eq. (7), we have the Drude weight as below

$$
D_{w}^{e}=\frac{e^{2}}{4 \pi m_{e}} \sum_{\lambda} \int d^{2} \mathbf{k}\left(k+\lambda k_{\alpha}\right)^{2} \cos ^{2} \theta \frac{\delta\left(k-k_{f}^{\lambda}\right)}{\left|k_{f}^{\lambda}+\lambda k_{\alpha}\right|},
$$

where $k_{f}^{\lambda}=-\lambda k_{\alpha}+\sqrt{2 \pi n_{e}-k_{\alpha}^{2}}$ are the spin-split Fermi wave-vectors. The final expression of the Drude weight is now

$$
D_{w}^{e}=\frac{\pi e^{2}}{m_{e}}\left(n_{e}-\frac{m_{e}^{2} \alpha_{e}^{2}}{2 \pi \hbar^{4}}\right)
$$

which is the same as given in Eq. (44) of Ref $\underline{36}$.
1 R. Winkler, Spin-orbit Coupling Effects in TwoDimensional Electron and Hole systems (Springer, Berlin 2003).

2 S. Bandyopadhyay and M. Cahay, Introduction to Spintronics (CRC press-2008).

3 I. Zutic, J. Fabian, and S. Das Sarma, Rev. Mod. Phys. 76, 323 (2004).

4 J. Fabian, A. Matos-Abiague, C. Ertler, P. Stano, and I. Zutic, Acta Physica Slovaca 57, 565 (2007).

${ }^{5}$ E. I. Rashba, Sov. Phys. Solid State 2, 1109 (1960).

6 Y. A. Bychkov and E. I. Rashba, J. Phys. C: Solid State Phys. 17, 6039 (1984).

7 J. M. Luttinger and W. Kohn, Phys. Rev. 97, 869 (1955).

8 G. Dresselhaus, Phys. Rev. 100, 580 (1955).

9 J. M. Luttinger, Phys. Rev. 102, 1030 (1956).

10 B. Das, D. C. Miller, S. Datta, R. Reifenberger, W. P. Hong, P. K. Bhattachariya, J. Sing, and M. Jaffe, Phys. Rev. B 39, 1411 (1989); J. Luo, H. Munekata, F. F. Fang, and P. J. Stiles, Phys. Rev. B 38, 10142 (1988); 41, 7685 (1990); J. Nitta, T. Akazaki, H. Takayanagi, and T. Enoki, Phys. Rev. Lett. 78, 1335 (1997).

11 F. Kuemmeth, S. Ilani, D. C. Ralph, and P. L. McEuen, Nature (London) 452, 448 (2008).

12 A. Varykhalov, J. Snchez-Barriga, A. M. Shikin, C. Biswas, E. Vescovo, A. Rybkin, D. Marchenko, and O. Rader Phys. Rev. Lett. 101, 157601 (2008).
13 D. Marchenko, J. Snchez-Barriga, M. R. Scholz, O. Rader, and A. Varykhalov Phys. Rev. B 87, 115426 (2013).

14 Y. J. Lin, K. Jimenez-Garcia, and I. B. Spielman, Nature 471, 83 (2011).

15 T. Ozawa and G. Baym, Phys. Rev. A 85, 013612 (2012).

16 E. I. Rashba and E. Ya. Sherman, Phys. Lett. A 129, 175 (1988).

17 R. Winkler, Phys. Rev. B 62, 4245 (2000).

18 R. Winkler, H. Noh, E. Tutuc, and M. Shayegan, Phys. Rev. B 65, 155303 (2002); G. M. Minkov, A. A. Sherstobitov, A. V. Germanenko, O. E. Rut, V. A. Larionova, and B. N. Zvonkov, Phys. Rev. B 71, 165312 (2005); F. Nichele, A. N. Pal, R. Winkler, C. Gerl, W. Wegscheider, T. Ihn, and K. Ensslin, Phys. Rev. B 89, 081306(R) (2014).

19 H. Nakamura, T. Koga, and T. Kimura, Phys. Rev. Lett. 108, 206601 (2012).

${ }^{20}$ R. Moriya, K. Sawano, Y. Hoshi, S. Masubuchi, Y. Shiraki, A. Wild, C. Neumann, G. Abstreiter, D. Bougeard, T. Koga, and T. Machida, Phys. Rev. Lett. 113, 086601 (2014).

21 D. W. Yuan, W. Xu, Z. Zeng, and F. Lu, Phys. Rev. B 72, 03320 (2005).

22 J. A. Maytorena, C. Lpez-Bastidas, and F. Mireles, Phys. Rev. B 74, 235313 (2006).

${ }^{23}$ M. Xie, G. Khalsa, and A. H. Macdonals, Phys. Rev. B 89, 245417 (2014). 
${ }^{24}$ C. H. Yang, W. Xu, Z. Zeng, F. Lu, and C. Zhang, Phys. Rev. B 74, 075321 (2006).

25 A. Wong and F. Mireles, Phys. Rev. B 81, 085304 (2010).

26 Z. Li, F. Marsiglio, and J. P. Carbotte, Sci. Rep. 3, 2828 (2013).

27 V. P. Gusynin, S. G. Sharapov, and J. P. Carbotte, Phys. Rev. B 75, 165407 (2007); T. Stauber, N. M. R. Peres, and A. K. Geim, Phys. Rev. B 78, 085432 (2008).

28 Z. Li and J. P. Carbotte, Phys. Rev. B 86, 205425 (2012); I. Miloevic, B. Nikolic, E. Dobardzic, M. Damnjanovic, I. Popov, and G. Seifert, Phys. Rev. B 76, 233414 (2007).

29 L. Stille, C. J. Tabert, and E. J. Nicol, Phys. Rev. B 86, 195405 (2012); L. Matthes, P. Gori, O. Pulci, and F. Bechstedt, Phys. Rev. B 87, 035438 (2013); C. J. Tabert and E. J. Nicol, Phys. Rev. B 87, 235426 (2013).

30 Z. Li and J. P. Carbotte, Phys. Rev. B 87, 155416 (2013); X. Xiao and W. Wen, Phys. Rev. B 88, 045442 (2013).

31 T. Ma and Q. Liu, Appl. Phys. Lett. 89, 112102 (2006).

${ }^{32}$ M. Zarea and S. E. Ulloa, Phys. Rev. B 73, 165306 (2006).

33 D. V. Bulaev and D. Loss, Phys. Rev. Lett. 95, 076805 (2005).

34 J. Schliemann and D. Loss, Phys. Rev. B 71, 085308 (2005).

35 N. W. Ashcroft and N. D. Mermin, Solid State Physics, (Harcourt College Publishes-2001).
36 A. Agarwal, S. Chesi, T. Jungwirth, J. Sinova, G. Vignale, and M. Polini, Phys. Rev. B 83, 115135 (2011).

37 M. Trushin and J. Schliemann, Phys. Rev. B 75, 155323 (2007); P. M. Krstaji, M. Pagano, and P. Vasilopoulos, Physica E: Low-dimensional Systems and Nanostructures 43, 893 (2011).

38 S. Shapira, U. Sivan, P. M. Solomon, E. Buchstab, M. Tischler, and G. Ben Yoseph, Phys. Rev. Lett. 77, 3181 (1996)).

39 J. Zhu, H. L. Stormer, L. N. Pfeiffer, K. W. Baldwin, and K. W. West, Phys. Rev. Lett. 90, 056805 (2003); E. Tutuc, S. Melinte, E. P. De Poortere, M. Shayegan, and R. Winkler, Phys. Rev. B 67, 241309(R) (2003).

40 R. Winkler, E. Tutuc, S. J. Papadakis, S. Melinte, M. Shayegan, D. Wasserman, and S. A. Lyon, Phys. Rev. B 72, 195321 (2005).

41 J. Schliemann, Phys. Rev. B 74, 045214 (2006).

42 S. Chesi and G. F. Giuliani, Phys. Rev. B 75, 155305 (2007).

43 T. Kernreiter, M. Governale, R. Winkler, and U. Zlicke, Phys. Rev. B 88, 125309 (2013).

44 C. Hamaguchi, Basic Semiconductor Physics, (SpringerVerlag-2010). 